\title{
Learning approaches used by students in an undergraduate emergency medical care programme
}

\author{
C Mosca, BTech Emergency Medical Care; A Makkink, BTech Emergency Medical Care, PDTE; \\ C Stein, BTech Emergency Medical Care, BTech Education (Post-School), PhD (Emergency Medicine)
}

Department of Emergency Medical Care, Faculty of Health Sciences, University of Johannesburg, South Africa

Corresponding author: C Stein (cstein@uj.ac.za)

Background. Students may primarily use either a deep learning approach (DLA) or surface learning approach (SLA) in response to their perceptions of the intrinsic and extrinsic factors within a given learning environment. By determining the learning approaches of students, one can provide important information on how they learn within an educational programme - information that can be used for various applications with regard to future structure and presentation of programme content.

Objectives. To determine which learning approaches (DLA or SLA) were being used by students in the Bachelor of Emergency Medical Care programme at the University of Johannesburg, South Africa, in each academic year of study. Further objectives were to determine which intrinsic and extrinsic factors influenced these choices and to assess whether learning approaches differed significantly between academic years of study.

Methods. This study was conducted using a quantitative design and a validated 20-question survey instrument. Data analysis was primarily descriptive, but also focused on whether there was a significant difference in learning approaches between the four years of study.

Results. Seventy students participated in the study, giving an overall response rate of $85 \%$. Results showed that most students predominantly used a DLA, and that there was no significant difference between the four academic years of study with regard to the predominant learning approach. More students appeared to be influenced to use a DLA by extrinsic factors than intrinsic factors.

Conclusion. Further research is needed to determine why students choose SLAs or DLAs, and the influence of the educational environment on this process. Afr J Health Professions Educ 2015;7(1):55-57. DOI:10.7196/AJHPE.393

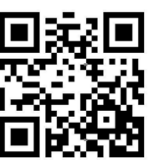

The term 'learning approaches' is used to describe what students do when engaging in the learning process, and what primarily motivates them to adopt a particular way of learning. Several studies have been conducted to determine the learning approaches that specific groups of students use, and the factors in the various groups that motivate or develop their adopted learning approaches. ${ }^{[1]}$ When defining learning approaches, there are two main categories, i.e. the deep learning approach (DLA) and surface learning approach (SLA). A consistent finding is that learning is a process and that approaches to learning are shaped by both intrinsic and extrinsic factors. ${ }^{[1]}$

\section{Process of learning}

Learning is a lifelong process that is a combination of three distinct variables: presage, process and product. Biggs ${ }^{[2]}$ conceptualised these variables into an interactive and interrelated system that can be used as a framework from which the process of learning can be understood.

The presage variable comprises factors that exist before the learning experience (those aspects that the student brings into the learning environment) and is a combination of intrinsic and extrinsic factors. Intrinsic factors consist of the personal and innate character traits of the student and can mean that a student is naturally hard working or not, dedicated or uncommitted, and represents the student's attitude in the learning process. ${ }^{[1]}$ Extrinsic factors consist of the learning environment into which the student enters and include the structure of the educational programme, prescribed workload, teaching methods and forms of assessment. ${ }^{[1,3]}$ Research has shown that the learning environment alone can push a student towards a DLA or an SLA. ${ }^{[1,2,4,5]}$
Learning as a process may be shaped by both intrinsic and extrinsic factors. ${ }^{[1]}$ DLAs are associated with the student engaging with, understanding and making personal meaning of the content being learnt. However, SLAs result in the student relying on memorisation and fact recall in a manner that leaves the information unrelated and isolated from the underlying meaning of the content learnt. DLAs are associated with 'knowledge transformation' and SLAs with 'information reproduction'. The primary distinction is that the SLA is generally relied on when the aim of learning is to momentarily perform well in an assessment. This does not reflect the level of competency achieved by the student in the tested subject matter; it is a reflection of their competency in being able to perform for assessments. The opposite is true for the DLA, where the aim of learning lies in the understanding and interpretation of the learning material, with performance in assessments being a secondary, or even consequent, aim. ${ }^{[2,5,6]}$

The Bachelor of Emergency Medical Care (BEMC) is a 4-year professional degree programme leading to registration with the Health Professions Council of South Africa as an Emergency Care Practitioner, the top tier of non-physician prehospital emergency care personnel. No formal study has yet been undertaken to determine student learning approaches and factors that may influence these in the BEMC programme. This knowledge gap makes it difficult for academic staff to understand how students are adapting to the learning content and environment, and limits understanding of whether students are applying appropriate learning strategies within each specific year of study. Additionally, insight regarding learning approaches may provide judgements to be made regarding the appropriateness of the BEMC programme structure and success of employed teaching methodologies. 


\section{Objectives}

The objective of this study was to determine what learning approaches (DLA or SLA) were adopted by students in each academic year of study and whether these differed significantly between years in a manner consistent with a progression from SLA to DLA between year 1 and year 4 .

The primary hypothesis was that first-year students would mostly use SLAs and that there would be a transition to an increasingly DLA from year 1 to year 4. Our motivations for this reasoning were based mostly on our anecdotal observations that first-year BEMC students are mainly poorly equipped to deal with a university-level academic workload and may therefore adopt an SLA to cope with passing assessments. Over time, and with academic progression from first to fourth year, we felt that this may change and a DLA may be adopted. We thought that the factors influencing students in this way would most likely be extrinsic and part of the university learning environment.

\section{Methods}

\section{Questionnaire}

The 20-item Biggs R-SPQ-2F questionnaire was used to assess the learning approach that BEMC students chose to adopt - DLA or SLA. ${ }^{[2]}$ The questionnaire was specifically designed as a sensitive tool to identify student approaches to learning (SAL) and has been previously validated. ${ }^{[7]}$

It also contains a sub-scaling system that allows data interpretation in a way that can identify the factors influencing the learning approach that students use. The motive subscale is used to identify the intrinsic factors that motivate students to adopt either a DLA or an SLA, and the strategy subscale to identify the extrinsic factors of the learning environment that motivate students in this regard. ${ }^{[2]}$

\section{Study sample and data collection}

All students registered for full-time study in the BEMC programme at the University of Johannesburg, South Africa, were invited to take part in the study. Those willing to participate and who signed the appropriate consent form were eligible to take part. Consenting students completed questionnaires before or immediately after scheduled lectures.

Ethical approval to conduct the study was obtained from the Faculty of Health Sciences Academic Ethics Committee at the University of Johannesburg.

\section{Data analysis}

Closed response data from completed questionnaires were captured onto a spreadsheet application and scored in accordance with the recommended scoring system. The main SAL scores were used to determine whether students used a DLA or an SLA. Answers for each specific approach were grouped and combined to reach an overall total. The group of questions with the highest total was considered indicative of the predominant learning approach of that particular respondent. Scores were grouped by academic year of study (first to fourth). Subscale data for surface and deep motive and strategy were also scored according to the questionnaire scoring system and grouped by academic year of study.

Differences in main SAL and subscale scores across academic years of study were compared using the Kruskal-Wallis test, as the data in each group were found to be non-normal. SPSS (version 17.0, SPSS Science, Chicago, USA) was used for inferential data analysis and all statistical tests were considered significant at $p<0.05$.

\section{Results}

Response rate data are shown in Table 1 . The decrease in student numbers from first to fourth year is due mainly to the fact that the BEMC programme was in the process of being phased in as a replacement for the previous National Diploma in Emergency Medical Care and did therefore not have a full complement of students in the third and fourth years.

The distribution of grouped main SAL scores across academic years is shown in Table 2. Groupings reflect the predominant approach to learning and participants were placed in either the DLA or SLA group based on their highest SAL score, as described above.

Almost three-quarters of participants across all academic years were classified as having a DLA and the remainder an SLA. A trend in proportional classification across the academic years, from first to fourth, can be seen in Table 2, with a progressive increase in those classified as having a DLA and a corresponding decrease in those with an SLA.

Results from analysis of the questionnaire subscales are shown in Table 3.

Table 1. Student responses by academic year

\begin{tabular}{llll}
\hline Academic year of study & Total responses, $\boldsymbol{n}$ & Registered students, $\boldsymbol{n}$ & Response rate, \% \\
\hline First year & 30 & 34 & 88 \\
Second year & 16 & 19 & 84 \\
Third year & 17 & 21 & 81 \\
Fourth year & 7 & 8 & 88 \\
Total & 70 & 82 & 85
\end{tabular}

Table 2. Distribution of main SAL score groupings across academic years

\begin{tabular}{lll}
\hline Academic year of study & Deep approach, $\boldsymbol{n}(\%)$ & Surface approach, $\boldsymbol{n}(\%)$ \\
\hline First year & $20(67)$ & $10(33)$ \\
Second year & $11(69)$ & $5(31)$ \\
Third year & $14(82)$ & $3(18)$ \\
Fourth year & $6(86)$ & $1(14)$ \\
Total & $51(73)$ & $19(27)$ \\
SAL = student approaches to learning. & &
\end{tabular}

Table 3. Distribution of SAL deep and surface subscale score groupings across academic years

\begin{tabular}{lllll}
\hline Academic year of study & $\begin{array}{l}\text { Deep motive, } \\
\boldsymbol{n}(\%)\end{array}$ & $\begin{array}{l}\text { Deep strategy, } \\
\boldsymbol{n}(\%)\end{array}$ & $\begin{array}{l}\text { Surface motive, } \\
\boldsymbol{n}(\%)\end{array}$ & $\begin{array}{l}\text { Surface strategy, } \\
\boldsymbol{n}(\%)\end{array}$ \\
\hline First year & $14(47)$ & $6(27)$ & 0 & $10(33)$ \\
Second year & $4(25)$ & $7(44)$ & 0 & $5(31)$ \\
Third year & $4(24)$ & $10(59)$ & 0 & $3(18)$ \\
Fourth year & $1(14)$ & $5(71)$ & 0 & $1(14)$ \\
Total & $23(45)$ & $28(55)$ & 0 & $19(100)$ \\
SAL = student approaches to learning. & & & &
\end{tabular}


Of the 51 participants classified as adopting a DLA, more were classified as adopting a deep strategy than being intrinsically motivated to follow a DLA. Furthermore, the proportion of participants falling into the deep motive category progressively decreased across academic years of study, while there is a corresponding increase in the proportion of participants classified as deep strategy. No participants in the SLA group indicated that they were motivated to do so by intrinsic factors.

Results of the Kruskal-Wallis test were used to test the null hypothesis that there was no difference in SAL main or subscale scores of learning approach across the four academic years of study (Table 4). No significant differences were found for any of the SAL scores.

\section{Discussion}

The results of this study show a large proportion of students adopting a DLA from their first academic year of study and this proportion increases further over the four years of study. These findings are in line with data from similar studies. This pattern has been seen in studies using either the Biggs SPQ instrument or the Entwistle-Ramsden Lancaster Approaches to Studying Inventory. ${ }^{[8-10]}$ However, the findings of the study by Martenson ${ }^{[11]}$ showed that an SLA among four cohorts of medical students was the predominant approach

About two-thirds of first-year participants were classified as using a DLA; this was unexpected and contrary to our hypothesis of a predominantly SLA in this group. We reasoned that the academic workload and transition from school to university in a cohort that we have observed over time as being relatively poorly prepared for university study would induce an SLA, if this had not already been the dominant approach for many participants at school.
Our results showed a $19 \%$ increase in the proportion of participants classified as adopting a DLA from first to fourth year (Table 2). This trend is in keeping with the literature, suggesting that many adult students tend to develop their use of DLA over time - from junior to more senior academic levels of study. In many cases, this appears to be intrinsically driven as students develop skills required to apply DLAs..$^{[1-3,7]}$

Subscale data on whether the primary motivation for the dominant learning approach was intrinsic or extrinsic offer some additional information regarding the participants in our study. Responses summarised in Table 3 suggest that roughly half of the participants in the first year of study were intrinsically motivated to adopt a DLA, i.e. they brought this learning approach with them into the learning environment. However, about one-quarter of participants in the same group were extrinsically motivated to adopt a DLA, suggesting that they did this in response to the academic demands of the learning environment in which they found themselves. ${ }^{[1,4,6]}$ Entwistle and Ramsden ${ }^{[12]}$ have suggested that the learning approach of students is ultimately influenced by the learning environment and that effective learning is the result of a unique combination of the student's preferred orientation to learning and the learning environment itself.

The progression over four academic years of study, with intrinsic motivation for a DLA becoming less prevalent and extrinsic motivation more prevalent (Table 3), suggests that the learning environment, including teaching methodologies and assessment practices, may become more of a driver to adopt a DLA. This is mirrored by the SLA subscale results, showing a decreasing prevalence of extrinsic motivation for participants to follow an SLA from first through to fourth year. No participants were identified as being intrinsically motivated to adopt an SLA.

Table 4. Results of the Kruskal-Wallis test: Difference in main student approaches to learning and subscale scores between academic years

\begin{tabular}{lllllll}
\hline & Deep & Surface & Deep motive & Deep strategy & Surface motive & Surface strategy \\
\hline$\chi^{2}$ & 2.626 & 3.154 & 0.301 & 6.696 & 1.384 & 4.493 \\
df & 3 & 3 & 3 & 3 & 3 & 3 \\
$p$ & 0.453 & 0.369 & 0.960 & 0.082 & 0.709 & 0.213 \\
df $=$ degrees of freedom. & & & &
\end{tabular}

Although our study provides valuable information on the adoption of SLAs and DLAs by students in our programme, more work is needed to understand in greater detail what drives these choices and in particular the role of the learning environment in this process. The proportional decrease across the four years of study in students adopting a deep motive (Table 3 ) in particular, is a trend of some importance for future investigation and monitoring over time. Future research in this area should be designed to follow one or more cohorts of students over the four years, which may clarify whether the trend observed in this study is still present longitudinally and, if so, whether any kind of intervention may be appropriate.

\section{Limitations}

The sample used for this study was relatively small, although it comprised a cross-sectional picture of almost the entire BEMC programme in our department at the time. The small number of fourth-year participants makes broader interpretation of the results difficult and there is a possibility that variance in this group may increase in future with increased size of the group. The small sample, and the very small fourth-year group size, increases the possibility of a Type II error in results of the inferential analysis presented in Table 4 .

\section{References}

1. Beattie V, Collins B, Mc Innes B. Deep and surface learning: A simple or simplistic dichotomy? Account Educ 1997;6(1):1-12.

2. Biggs J, Kember D, Leung DYP. The Revised Two Factor Study Process Questionnaire: R-SPQ-2F. Br J Educ Psychol 2001;71:133-149.

Alkadhi S. Learning Theory: Adult Education: Andragogy. [Dissertation] Monterey Bay, Calif.: California State University, 2008.

4. Ames C. Classrooms: Goals, structures, and student motivation. Educ Psychol 1992;84(3):261-271. [http://dx.doi.org/10.1037/00220663.84.3.261]

Ramsden P. The context of learning in academic departments. In Marton F, Hounsell D, Entwistle N. The Experience of Learning: Implications for Teaching and Studying in Higher Education. 3rd ed. Edinburgh: University of Edinburgh, Centre for Teaching, Learning and Assessment, 2005:198-213.

6. Entwistle N. Promoting deep learning through teaching and assessment: Conceptual frameworks and educational contexts. TLRP Conference, Leicester, November 2000. http://www.ed.ac.uk/etl/docs. entwistle.pdf (accessed 8 October 2013).

7. Lizzio A, Wilson K, Simons R. University students' perceptions of the learning environment and academic outcomes: Implications for theory and practice. Stud High Educ 2002;27(1):27-52. [http://dx.doi org/10.1080/03075070120099359]

8. Newble DI, Gordon MI. The learning style of medical students. Med Educ 1985;19(1):3-8.

9. Emilia $\mathrm{O}$, Mulholland $\mathrm{H}$. Approaches to learning of students in an Indonesian . Emilia O, Mulholland H. Approaches to learning of
medical school. Med Educ 1991;25(6):462-470.

10. Mattick K, Knight L. The importance of vocational and social aspects of approaches to learning for medical students. Adv Health Sci Educ Theory Pract 2009;14(5):629-644.

11. Martenson D. Students' approaches to studying in four medical schools. Med Educ 1986;20:532-534.

12. Entwistle N, Ramsden P. Understanding Student Learning. London: Croom Helm, 1983:209. 